Kragujevac Journal of Mathematics

Volume 44(2) (2020), Pages 299-312.

\title{
HYERS-ULAM STABILITY OF A FREE AND FORCED VIBRATIONS
}

\author{
R. MURALI ${ }^{1}$ AND A. PONMANA SELVAN ${ }^{1}$
}

\begin{abstract}
In this paper, we study the Hyers-Ulam stability and Hyers-UlamRassias stability of the general differential equation of the Free Damped Vibrations, Undamped Vibrations and Forced Vibrations by using initial conditions.
\end{abstract}

\section{INTRODUCTION}

In every day life we come across numerous things that move. These motions are of two types, viz (i) the motion in wich the body moves about a mean position or a fixed point an (ii) the motion in which the body moves from one place to another place with respect to time. The first type of motion of a body about a mean position is called oscillatory motion. A moving train, flying aeroplane and moving ball etc., corresponds to the second type of motion. An oscillating pendulum, vibrations of a stretched string, movement of water in a cup, vibration of electrons are the examples of oscillatry motions. Vibration and oscillation are part of our every day life. With in a minute of waking up, we may well experience vibrations in a wide variety of forms: the buzzing of the alarm clock; the bounce of a bed; the oscillation of a loud speaker, electric tooth brush or an electric razor, musical instruments, machinery and traffic, noisy and annoying. A common feature of many of these vibrations of oscillations is that the motion is repetitive of periodic. Such a motions are called periodic motions. In nature, all these mechanical vibrations are Simple Harmonic Motion.

The theory of stability is an important branch of the qualitative theory of differential equations. In 1940, Ulam [28] posed a problem concerning the stability of functional

Key words and phrases. Hyers-Ulam stability, Hyers-Ulam-Rassias stability, free damped vibrations, undamped vibrations, forced vibrations and initial conditions.

2010 Mathematics Subject Classification. Primary: 34K20, 26D10. Secondary: 34A30, 34A40, 39B82, 44A10, 39A10, 34C20, 45D05.

DOI 10.46793/KgJMat2002.299M

Received: March 31, 2018.

Accepted: April 24, 2018. 
equation: "Give conditions in order for a linear function near an approximately linear function to exist." A year later, Hyers [11] gave an answer to the problem of Ulam for additive functions defined on Banach spaces. Thereafter, Aoki [3], Bourgin [4] and Rassias [21] improved the result reported in [11]. After that, many mathematicians have extended the Ulam's problem to other functional equations on various spaces in different directions (see $[1,5-8,12,15,24,25]$ ).

Definitions of both Hyers-Ulam stability and Hyers-Ulam-Rassias stability have applicable significance since it means that if one is studying an Hyers-Ulam stable or Hyers-Ulam-Rassias stable system then one does not have to reach the exact solution. (Which is usually is quite difficult or time consuming). This is quite useful in many applications. For example, numerical analysis, optimization, biology, economics, dynamic programming, wireless sensor networks, physics, chemistry, geometry and etc., where finding the exact solution is quite difficult.

A generalization of Ulam's problem was recently proposed by replacing functional equations with differential equations: The differential equation

$$
\phi\left(f, x, x^{\prime}, x^{\prime \prime}, \ldots, x^{(n)}\right)=0
$$

has the Hyers-Ulam stability if for a given $\epsilon>0$ and a function $x$ such that $\left|\phi\left(f, x, x^{\prime}, x^{\prime \prime}, \ldots, x^{(n)}\right)\right| \leq \epsilon$, there exists a solution $x_{a}$ of the differential equation such that $\left|x(t)-x_{a}(t)\right| \leq K(\epsilon)$ and $\lim _{\epsilon \rightarrow 0} K(\epsilon)=0$.

Oblaza seems to be the first author who has investigated the Hyers-Ulam stability of linear differential equations (see [19,20]). Thereafter, Alsina and Ger published their paper [2], which handles the Hyers-Ulam stability of the linear differential equation $y^{\prime}(t)=y(t)$. Those previous results were extended to the Hyers-Ulam stability of linear differential equations of first order and higher orders in $[9,10,13,14,16-18,22$, $23,26,27,29,30]$.

These days, the Hyers-Ulam stability of differential equation is investigated and the investigation is ongoing. Motivated and connected by above results, we prove the Hyers-Ulam stability of the general differential equation of Free Damped Vibrations (FDV),

$$
x^{\prime \prime}(t)+2 l x^{\prime}(t)+k^{2} x(t)=0,
$$

Undamped Vibrations (UV)

$$
x^{\prime \prime}(t)+k^{2} x(t)=0
$$

and Forced Vibrations (FV)

$$
x^{\prime \prime}(t)+2 l x^{\prime}(t)+k^{2} x(t)=L \sin p t,
$$

by using initial conditions

$$
x(a)=x^{\prime}(a)=0,
$$

where $x(t) \in C^{2}(I), I=[a, b] \subseteq \mathbb{R}$. 


\section{Preliminaries}

Simple Harmonic Motion plays an important role and it is the one of the most important example of periodic motion. Clock, car shock absorbers, musical instru ments, bungee jumping, diving board, the process of hearing, earth quake, proof building metronome, a swing, a rocking chair, clock pendulum, heart beat and breathing these are the some common examples of Simple Harmonic Motion in real life, but there are countless more applications.

Simple Harmonic Motion. When a body moves such that its acceleration is always directed towards a certain fixed point and varies directly as its distance from that point, the body is said to execute harmonic motion.

For such a motion to take place the force acting on the body should be directed towards the fixed point and should also be proportional to the displacement, i.e., the distance from the fixed point. The function of the force is to bring the body back to its equilibrium position and hence this force is often known as restoring force.

Consider a particle of mass $m$ executing simple harmonic motion. If $x$ be the displacement of the particle from equilibrium position at any instant $t$, the resulting force $F$ acting on the particle is given by $F \propto x$ (or) $F=-s x$, where $s$ is the force constant of proportionality or stiffness. The negative sign is used to indicate that the direction of the force is opposite to the direction of increasing displacement.

If $\frac{d^{2} x}{d t^{2}}$ is the acceleration of the particle at time $t$, then

$$
m \frac{d^{2} x}{d t^{2}}=-s x(\text { or }) \frac{d^{2} x}{d t^{2}}+\frac{s}{m} x=0
$$

substituting $k^{2}=\frac{s}{m}$, we get $\frac{d^{2} x}{d t^{2}}+k^{2} x=0$. This is the general differential equation of motion of a simple harmonic oscillator.

Free Vibrations. When the bob of a simple pendulum (in vacuum) is displaced from its mean position and left, it executes simple harmonic motion. The time period of oscillation depends only on the length of the pendulum and the acceleration due to the gravity at the place. The pendulum will continue to oscillate with the same time periodic and amplitude for any length of time. In such cases there is no loss of energy by friction or otherwise. In all similar cases, the vibrations will be undamped free vibrations. The amplitude swing remains constant.

Damping. The amplitude of a vibrating string, a sounding tuning fork and an oscillating pendulum goes on gradually decreasing and ultimately these bodies stop vibrating. It is because some energy is inevitably lost due to resistive or viscous forces. For example, in the case of a simple pendulum, energy is lost due to friction at the supports and resistance of air. The resistance offered by a damping force is known as damping. When the damping is small it does not produce any significant change 
in the undamped motion of the vibrating body. In such a case, the damping force is proportional to the velocity of the vibrating body.

Damped vibrations. In actual particle, when the pendulum vibrates in air medium, there are frictional forces and consequently energy is dissipated in each vibration. The amplitude of swing decreases continuously with time and finally the oscillations die out. Such vibrations are called free damped vibrations. The dissipated energy appears as heat either within the system itself or in the surrounding medium. The dissipative force due to friction etc. (resistance in $L C R$ circuit) is proportional to the velocity of the particle at that instant. That is, in an ideal simple harmonic motion, the displacement follows a sine curve for an infinite time. This is because the total energy remains constant. In actual practice, the simple harmonic oscillator always experiences frictional or resistive forces due to which some energy is lost and the oscillations get damped. The amplitude of vibration decreases gradually an ultimately the body comes to rest.

The decay of amplitude with time is called damping. Those simple harmonic vibrations where amplitude decreases with the passage of time are called damped simple harmonic vibrations.

Let $\mu \frac{d x}{d t}$ be the dissipative force due to friction or other phenomenon. Therefore, the differential equation in the case of Free Damped Vibration is,

$$
m \frac{d^{2} x}{d t^{2}}+T x+\mu \frac{d x}{d t}=0
$$

or

$$
\frac{d^{2} x}{d t^{2}}+\left(\frac{\mu}{m}\right) \frac{d x}{d t}+\left(\frac{T}{m}\right) x=0
$$

The above equation is similar to a general differential equation of the form

$$
\frac{d^{2} x}{d t^{2}}+2 l \frac{d x}{d t}+k^{2} x=0
$$

or

$$
x^{\prime \prime}(t)+2 l x^{\prime}(t)+k^{2} x(t)=0,
$$

where $k^{2}=\frac{T}{m}, l$ is known as the damping coefficient and $2 l$ gives the force due to resistance of the medium per unit mass per unit velocity. Equation (2.2) is known as differential equation of damped simple harmonic motion. Also, it can be written in the form of $x^{\prime \prime}(t)+a x^{\prime}(t)+b x(t)=0$ whose solution is,

$$
x(t)= \begin{cases}e^{\left(\frac{-1}{2} a t\right)}\left[c_{1} e^{\left(\frac{1}{2} \lambda t\right)}+c_{2} e^{\left(\frac{-1}{2} \lambda t\right)}\right], & \text { if } \lambda^{2}=a^{2}-4 b>0, \\ e^{\left(\frac{-1}{2} a t\right)}\left[c_{1} \sin \left(\frac{1}{2} \lambda t\right)+c_{2} \cos \left(\frac{1}{2} \lambda t\right)\right], & \text { if } \lambda^{2}=4 b-a^{2}>0, \\ e^{\left(\frac{-1}{2} a t\right)}\left[c_{1} x+c_{2}\right], & \text { if } a^{2}=4 b .\end{cases}
$$


Undamped Vibrations. For a simple harmonic vibrating particle, the kinetic energy for displacement $x$ is given by $\frac{1}{2} m\left(\frac{d x}{d t}\right)^{2}$. At the same instant, the potential energy of the particle is $\frac{1}{2} K x^{2}$, where $K$ is the restoring force per unit displacement. The differential equation in the case of undamped free vibrations is,

$$
m \frac{d^{2} x}{d t^{2}}+T x=0 \text { or } \frac{d^{2} x}{d t^{2}}+\left(\frac{T}{m}\right) x=0
$$

it takes the form

$$
x^{\prime \prime}(t)+k^{2} x(t)=0,
$$

where $k^{2}=\left(\frac{T}{m}\right)$. This is only an ideal case. Here it has been assumed that the vibrations are free and undamped.

Forced Vibrations. The time period of a body executing simple harmonic motion depends on the dimension of the body and its elastic properties The vibrations of such a body die out with time due to dissipation of energy. If some external periodic force is constantly applied on the body, the body continues to oscillate under the influence of such external force. Such vibrations of the body are called forced vibrations.

Initially, the amplitude of the swing increases, then decreases with time, becomes minimum and again increases. This will be repeated if the external periodic force is constantly applied on the system. In such cases the body will finally be forced to vibrate with the same frequency as that of the applied force. The frequency of the forced vibration is different from the natural frequency of vibration of the body. The amplitude of the forced vibration of the body depends on the difference between the natural frequency and the frequency applied force. The amplitude will be large of difference in frequency is small and vice versa. For forced vibrations, equation (2.1) is modified in the form

$$
\begin{gathered}
m \frac{d^{2} x}{d t^{2}}+T x+\mu \frac{d x}{d t}=F \sin p t, \\
x^{\prime \prime}(t)+2 l x^{\prime}(t)+k^{2} x(t)=L \sin p t .
\end{gathered}
$$

Here $p$ is angular frequency of the applied periodic force and $L=\left(\frac{F}{m}\right)$.

Now, we give the definition of Hyers-Ulam stability and Hyers-Ulam-Rassias stability of the general differential equation of Free Damped Vibrations (FDV) (1.1), Undamped Vibrations (UV) (1.2) and Forced Vibrations (FV) (1.3) by using initial conditions (1.4).

Definition 2.1. We say that the general differential equation of Free Damped Vibrations (1.1) has the Hyers-Ulam stability with initial conditions (1.4), if there exists a positive constant $K$ with the following property: For every $\epsilon>0, x \in C^{2}(I)$ satisfies the inequality

$$
\left|x^{\prime \prime}(t)+2 l x^{\prime}(t)+k^{2} x(t)\right| \leq \epsilon
$$


then there exists some $y \in C^{2}(I)$ satisfies $y^{\prime \prime}(t)+2 l y^{\prime}(t)+k^{2} y(t)=0$ with initial conditions $y(a)=y^{\prime}(a)=0$ sucht that $|x(t)-y(t)| \leq K \epsilon$. We call such $K$ as a Hyers-Ulam stability constant for the differential equation of Free Damped Vibrations (1.1) with initial conditions (1.4).

Definition 2.2. We say that the general differential equation of Free Damped Vibrations (1.1) has the Hyers-Ulam-Rassias stability with $\phi(\cdot)$, where $\phi: \mathbb{R} \rightarrow[0, \infty)$ and initial conditions (1.4), if there exists a positive constant $K$ with the following property: If $x \in C^{2}(I)$ is such that satisfying the inequality

$$
\left|x^{\prime \prime}(t)+2 l x^{\prime}(t)+k^{2} x(t)\right| \leq \phi(t),
$$

then there exists some $y \in C^{2}(I)$ satisfying $y^{\prime \prime}(t)+2 l y^{\prime}(t)+k^{2} y(t)=0$ with initial conditions $y(a)=y^{\prime}(a)=0$ such that $|x(t)-y(t)| \leq K \phi(t)$. We call such $K$ as a Hyers-Ulam-Rassias stability constant for the differential equation of Free Damped Vibrations (1.1) with initial conditions (1.4).

Definition 2.3. We say that the general differential equation of Undamped Vibrations (1.2) has the Hyers-Ulam stability with initial conditions (1.4), if there exists a positive constant $K$ with the following property: for every $\epsilon>0, x \in C^{2}(I)$ satisfying the inequality

$$
\left|x^{\prime \prime}(t)+k^{2} x(t)\right| \leq \epsilon,
$$

then there exists some $y \in C^{2}(I)$ satisfying the differential equation $y^{\prime \prime}(t)+k^{2} y(t)=0$ with initial conditions $y(a)=y^{\prime}(a)=0$ sucht that $|x(t)-y(t)| \leq K \epsilon$. We call such $K$ as a Hyers-Ulam stability constant for the differential equation of Undamped Vibrations (1.2) with initial conditions (1.4).

Definition 2.4. We say that the general differential equation of Undamped Vibrations (1.2) has the Hyers-Ulam-Rassias stability with $\phi(\cdot)$, where $\phi: \mathbb{R} \rightarrow[0, \infty)$ and initial conditions (1.4), if there exists a positive constant $K$ with the following property: If $x \in C^{2}(I)$ is such that satisfying the inequality

$$
\left|x^{\prime \prime}(t)+k^{2} x(t)\right| \leq \phi(t),
$$

then there exists some $y \in C^{2}(I)$ satisfying the differential equation $y^{\prime \prime}(t)+k^{2} y(t)=0$ with initial conditions $y(a)=y^{\prime}(a)=0$ sucht that $|x(t)-y(t)| \leq K \phi(t)$. We call such $K$ as a Hyers-Ulam-Rassias stability constant for the differential equation of Undamped Vibrations (1.2) with initial conditions (1.4).

Definition 2.5. We say that the general differential equation of Forced Vibrations (1.3) has the Hyers-Ulam stability with initial conditions (1.4), if there exists a positive constant $K$ with the following property: For every $\epsilon>0, x \in C^{2}(I)$ satisfying

$$
\left|x^{\prime \prime}(t)+2 l x^{\prime}(t)+k^{2} x(t)-L \sin p t\right| \leq \epsilon,
$$

then there exists some $y \in C^{2}(I)$ satisfies the differential equation

$$
y^{\prime \prime}(t)+2 l y^{\prime}(t)+k^{2} y(t)=L \sin p t,
$$


with initial conditions $y(a)=y^{\prime}(a)=0$ sucht that $|x(t)-y(t)| \leq K \epsilon$. We call such $K$ as a Hyers-Ulam stability constant for the differential equation of Forced Vibrations (1.3) with initial conditions (1.4).

Definition 2.6. We say that the general differential equation of Forced Vibrations (1.3) has the Hyers-Ulam-Rassias stability with $\phi(\cdot)$, where $\phi: \mathbb{R} \rightarrow[0, \infty)$ and initial conditions (1.4), if there exists a positive constant $K$ with the following property: If $x \in C^{2}(I)$ is such that satisfies

$$
\left|x^{\prime \prime}(t)+2 l x^{\prime}(t)+k^{2} x(t)-L \sin p t\right| \leq \phi(t)
$$

then there exists some $y \in C^{2}(I)$ satisfying the differential equation

$$
y^{\prime \prime}(t)+2 l y^{\prime}(t)+k^{2} y(t)=L \sin p t,
$$

with initial conditions $y(a)=y^{\prime}(a)=0$ such that $|x(t)-y(t)| \leq K \phi(t)$. We call such $K$ as a Hyers-Ulam-Rassias stability constant for the differential equation of Forced Vibrations (1.3) with initial conditions (1.4).

\section{Hyers-Ulam Stability}

In this section, we prove the Hyers-Ulam stability of a general differential equation of FDV (1.1), UV (1.2) and FV (1.3) by using initial conditions (1.4). Now, we prove the Hyers-Ulam stability of the general differential equation of FDV (1.1) by using the initial conditions (1.4).

Theorem 3.1. If $x: I \rightarrow \mathbb{R}$ be a twice continuously differentiable function such that $\left|x^{\prime}(t)\right| \leq|x(t)|$ satisfies the differential inequality

$$
\left|x^{\prime \prime}(t)+2 l x^{\prime}(t)+k^{2} x(t)\right| \leq \epsilon
$$

with initial conditions (1.4) then the differential equation (1.1) has the Hyers-Ulam stability.

Proof. For every $\epsilon>0$, there exists $x \in C^{2}(I)$ such that $\left|x^{\prime}(t)\right| \leq|x(t)|$ satisfies the differential inequality (3.1) with initial conditions (1.4) and define $M=\max _{t \in I}|x(t)|$. Then by the inequality (3.1), we have

$$
-\epsilon \leq x^{\prime \prime}(t)+2 l x^{\prime}(t)+k^{2} x(t) \leq \epsilon,
$$

multiplying the above inequality by $x^{\prime}(t)$ and then integrate, we obtain that

$$
\begin{aligned}
& \int_{a}^{t}-\epsilon x^{\prime}(\tau) d \tau \leq \int_{a}^{t}\left\{x^{\prime \prime}(\tau)+2 l x^{\prime}(\tau)+k^{2} x(\tau)\right\} x^{\prime}(\tau) d \tau \leq \int_{a}^{t} \epsilon x^{\prime}(\tau) d \tau \\
& -\epsilon \int_{a}^{t} x^{\prime}(\tau) d \tau \leq \int_{a}^{t} x^{\prime \prime}(\tau) x^{\prime}(t) d \tau+2 l \int_{a}^{t} x^{\prime}(\tau)^{2} d \tau+k^{2} \int_{a}^{t} x(\tau) x^{\prime}(\tau) d \tau \leq \epsilon \int_{a}^{t} x^{\prime}(\tau) d \tau,
\end{aligned}
$$




$$
-2 \epsilon x(t) \leq x^{\prime}(t)^{2}+k^{2} x(t)^{2}+4 l \int_{a}^{t} x^{\prime}(\tau)^{2} d \tau \leq 2 \epsilon x(t),
$$

from which we get that

$$
\begin{aligned}
k^{2} x(t)^{2} & \leq 2 \epsilon x(t)-4 l \int_{a}^{t} x^{\prime}(\tau)^{2} d \tau, \\
M^{2} & \leq \frac{2 \epsilon}{k^{2}} M+\frac{4 l}{k^{2}} M^{2}(b-a) .
\end{aligned}
$$

Choose $\gamma=\frac{4 l(b-a)}{k^{2}}$, we get

$$
M \leq \frac{2 \epsilon}{k^{2}(1-\gamma)}
$$

which gives $|x(t)| \leq \frac{2 \epsilon}{k^{2}(1-\gamma)}$. Hence, $|x(t)| \leq K \epsilon$, where $K=\frac{2}{k^{2}(1-\gamma)}$. It is easy to see that, $y\left(t_{0}\right) \equiv 0$ is a solution of $x^{\prime \prime}(t)+2 l x^{\prime}(t)+k^{2} x(t)=0$ with initial condition (1.4) such that $\left|x(t)-y\left(t_{0}\right)\right| \leq K \epsilon$. Hence, by the virtue of Definition 2.1, the differential equation of FDV (1.1) has the Hyers-Ulam stability.

Now, we prove the Hyers-Ulam stability of the general differential equation of UV (1.2) with initial conditions (1.4).

Theorem 3.2. If $x: I \rightarrow \mathbb{R}$ be a twice continuously differentiable function satisfies the inequality

$$
\left|x^{\prime \prime}(t)+k^{2} x(t)\right| \leq \epsilon
$$

with initial conditions (1.4) then the differential equation (1.2) has the Hyers-Ulam stability.

Proof. For every $\epsilon>0$, there exists $x \in C^{2}(I)$, satisfies the differential inequality (3.2) with initial conditions (1.4) and define $M=\max _{t \in I}|x(t)|$. Then by the inequality (3.2), we have

$$
-\epsilon \leq x^{\prime \prime}(t)+k^{2} x(t) \leq \epsilon
$$

multiplying the above inequality by $x^{\prime}(t)$ and then integrating $a$ to $t$, we get

$$
\begin{aligned}
-\epsilon \int_{a}^{t} x^{\prime}(\tau) d \tau & \leq \int_{a}^{t}\left\{x^{\prime \prime}(\tau)+k^{2} x(\tau)\right\} x^{\prime}(\tau) d \tau \leq \epsilon \int_{a}^{t} x^{\prime}(\tau) d \tau, \\
-2 \epsilon x(t) & \leq x^{\prime}(t)^{2}+k^{2} x(t)^{2} \leq 2 \epsilon x(t)
\end{aligned}
$$

from which we obtain that $k^{2} x(t)^{2} \leq 2 \epsilon x(t)$. Hence, $|x(t)| \leq K \epsilon$, where $K=\frac{2}{k^{2}}$. It is easy to see that, $y\left(t_{0}\right) \equiv 0$ is a solution of $x^{\prime \prime}(t)+k^{2} x(t)=0$ with initial condition (1.4) such that

$$
\left|x(t)-y\left(t_{0}\right)\right| \leq K \epsilon .
$$

Hence by the virtue of Definition 2.3, the differential equation of UV (1.2) has the Hyers-Ulam stability. 
Finally, in this section we would like to prove the Hyers-Ulam stability of the general differential equation of FV (1.3) with initial conditions (1.4).

Theorem 3.3. If $x: I \rightarrow \mathbb{R}$ be a twice continuously differentiable function such that $\left|x^{\prime}(t)\right| \leq|x(t)|$ satisfies the differential inequality

$$
\left|x^{\prime \prime}(t)+2 l x^{\prime}(t)+k^{2} x(t)-L \sin p t\right| \leq \epsilon
$$

with initial conditions (1.4) then the differential equation (1.3) has the Hyers-Ulam stability.

Proof. For every $\epsilon>0$, there exists $x \in C^{2}(I)$ such that $\left|x^{\prime}(t)\right| \leq|x(t)|$ satisfies the differential inequality (3.3) with initial conditions (1.4) and define $M=\max _{t \in I}|x(t)|$. Then by the inequality (3.3), we have

$$
-\epsilon \leq x^{\prime \prime}(t)+2 l x^{\prime}(t)+k^{2} x(t)-L \sin p t \leq \epsilon .
$$

Multiplying the above inequality by $x^{\prime}(t)$ and then integrate, we obtain that

$$
\begin{aligned}
\int_{a}^{t}-\epsilon x^{\prime}(\tau) d \tau & \leq \int_{a}^{t}\left\{x^{\prime \prime}(\tau)+2 l x^{\prime}(\tau)+k^{2} x(\tau)-L \sin p \tau\right\} x^{\prime}(\tau) d \tau \leq \int_{a}^{t} \epsilon x^{\prime}(\tau) d \tau \\
-\epsilon \int_{a}^{t} x^{\prime}(\tau) d \tau & \leq \int_{a}^{t} x^{\prime \prime}(\tau) x^{\prime}(\tau) d \tau+2 l \int_{a}^{t} x^{\prime}(\tau)^{2} d \tau+k^{2} \int_{a}^{t} x(\tau) x^{\prime}(\tau) d \tau \\
-L \int_{a}^{t} x^{\prime}(\tau) \sin p \tau d \tau & \leq \epsilon \int_{a}^{t} x^{\prime}(\tau) d \tau, \\
-2 \epsilon x(t) & \leq x^{\prime}(t)^{2}+k^{2} x(t)^{2}+4 l \int_{a}^{t} x^{\prime}(\tau)^{2} d \tau-2 L \int_{a}^{t} x^{\prime}(\tau) \sin p \tau d \tau \\
& \leq 2 \epsilon x(\tau),
\end{aligned}
$$

from which we get that

$$
\begin{aligned}
k^{2} x(t)^{2} & \leq 2 \epsilon x(t)-4 l \int_{a}^{t} x^{\prime}(\tau)^{2} d \tau+2 L \int_{a}^{t} x^{\prime}(\tau) \sin p \tau d \tau, \\
M^{2} & \leq \frac{2 \epsilon}{k^{2}} M+\frac{4 l}{k^{2}} M^{2}(b-a)+\frac{2 L M}{k^{2}} \sin p a .
\end{aligned}
$$

Choose $\gamma=\frac{4 l(b-a)}{k^{2}}$, we get $M \leq \frac{2 \epsilon+2 L \sin p a}{k^{2}(1-\gamma)}$, which gives

$$
|x(t)| \leq \frac{2 \epsilon+2 L \sin p a}{k^{2}(1-\gamma)} .
$$

Hence $|x(t)| \leq K(\epsilon)$, where $K(\epsilon)=\frac{2 \epsilon+2 L \sin p a}{k^{2}(1-\gamma)}$ for all $t \in I$. Obviously, $y\left(t_{0}\right) \equiv 0$ is a solution of the differential equation $x^{\prime \prime}(t)+2 l x^{\prime}(t)+k^{2} x(t)-L \sin p t$ with (1.4) such that $\left|x(t)-y\left(t_{0}\right)\right| \leq K(\epsilon)$. 
Hence by the virtue of Definition 2.5, the differential equation of FV (1.3) has the Hyers-Ulam stability.

\section{Hyers-Ulam-Rassias Stability}

In this section, we prove the Hyers-Ulam-Rassias stability of a general differential equation of FDV (1.1), UV (1.2) and FV (1.3) by using initial conditions (1.4). Now, we prove the Hyers-Ulam-Rassias stability of the general differential equation of FDV (1.1) by using the initial conditions (1.4).

Theorem 4.1. If $x: I \rightarrow \mathbb{R}$ be a twice continuously differentiable function such that $\left|x^{\prime}(t)\right| \leq|x(t)|$ with $\phi(t)$, where $\phi: \mathbb{R} \rightarrow[0, \infty)$ satisfies the differential inequality

$$
\left|x^{\prime \prime}(t)+2 l x^{\prime}(t)+k^{2} x(t)\right| \leq \phi(t),
$$

with initial conditions (1.4) then the differential equation (1.1) has the Hyers-UlamRassias stability with respect to $\phi(t)$ such that $\phi(a)=0$.

Proof. If $x \in C^{2}(I)$ is such that $\left|x^{\prime}(t)\right| \leq|x(t)|$ with $\phi: \mathbb{R} \rightarrow[0, \infty)$ satisfies the differential inequality (4.1) with initial conditions (1.4) and define $M=\max _{t \in I}|x(t)|$. Then by the inequality (4.1), we have

$$
-\phi(t) \leq x^{\prime \prime}(t)+2 l x^{\prime}(t)+k^{2} x(t) \leq \phi(t)
$$

multiplying the above inequality by $x^{\prime}(t)$ and then integrating with the limits $a$ to $t$, we obtain that

$$
\begin{gathered}
\int_{a}^{t}-\phi(\tau) x^{\prime}(\tau) d \tau \leq \int_{a}^{t}\left\{x^{\prime \prime}(\tau)+2 l x^{\prime}(\tau)+k^{2} x(\tau)\right\} x^{\prime}(\tau) d \tau \leq \int_{a}^{t} \phi(\tau) x^{\prime}(\tau) d \tau, \\
-2 \int_{a}^{t} x^{\prime}(\tau) \phi(\tau) d \tau \leq x^{\prime}(t)^{2}+k^{2} x(t)^{2}+4 l \int_{a}^{t} x^{\prime}(\tau)^{2} d \tau \leq 2 \int_{a}^{t} x^{\prime}(\tau) \phi(\tau) d \tau,
\end{gathered}
$$

from which we get that

$$
\begin{aligned}
k^{2} x(t)^{2} & \leq 2 \int_{a}^{t} x^{\prime}(\tau) \phi(\tau) d \tau-4 l \int_{a}^{t} x^{\prime}(\tau)^{2} d \tau, \\
M^{2} & \leq \frac{2}{k^{2}} M \phi(t)+\frac{4 l}{k^{2}} M^{2}(b-a) .
\end{aligned}
$$

Choose $\gamma=\frac{4 l(b-a)}{k^{2}}$, we get

$$
M \leq \frac{2 \phi(t)}{k^{2}(1-\gamma)}
$$

which gives $|x(t)| \leq \frac{2 \phi(t)}{k^{2}(1-\gamma)}$. Hence, $|x(t)| \leq K \phi(t)$ for all $t \in I$, where $K=\frac{2}{k^{2}(1-\gamma)}$. Obviously, $y\left(t_{0}\right) \equiv 0$ is a solution of the differential equation (1.1) with (1.4) such that

$$
\left|x(t)-y\left(t_{0}\right)\right| \leq K \phi(t)
$$


Hence by the virtue of Definition 2.2, FDV (1.1) has the Hyers-Ulam-Rassias stability with initial conditions (1.4).

Now, we are going to prove the Hyers-Ulam-Rassias stability of the differential equation of UV (1.2) with initial conditions (1.4).

Theorem 4.2. If $x: I \rightarrow \mathbb{R}$ be a twice continuously differentiable function such that $\left|x^{\prime}(t)\right| \leq|x(t)|$ with $\phi(t)$, where $\phi: \mathbb{R} \rightarrow[0, \infty)$ satisfies the differential inequality

$$
\left|x^{\prime \prime}(t)+k^{2} x(t)\right| \leq \phi(t)
$$

with initial conditions (1.4) then the general differential equation of $U V$ (1.2) has the Hyers-Ulam-Rassias stability with respect to $\phi(t)$ such that $\phi(a)=0$.

Proof. If $x \in C^{2}(I)$ is such that $\left|x^{\prime}(t)\right| \leq|x(t)|$ with $\phi: \mathbb{R} \rightarrow[0, \infty)$ satisfies the differential inequality (4.2) with initial conditions (1.4) and define $M=\max _{t \in I}|x(t)|$. Then by the inequality $(4.2)$, we have

$$
-\phi(t) \leq x^{\prime \prime}(t)+k^{2} x(t) \leq \phi(t),
$$

multiplying the above inequality by $x^{\prime}(t)$ and then integrating $a$ to $t$, we get

$$
\begin{aligned}
& -\int_{a}^{t} x^{\prime}(\tau) \phi(\tau) d \tau \leq \int_{a}^{t}\left\{x^{\prime \prime}(\tau)+k^{2} x(\tau)\right\} x^{\prime}(\tau) d \tau \leq \int_{a}^{t} x^{\prime}(\tau) \phi(\tau) d \tau, \\
& -2 \int_{a}^{t} x^{\prime}(\tau) \phi(\tau) d \tau \leq x^{\prime}(t)^{2}+k^{2} x(t)^{2} \leq 2 \int_{a}^{t} x^{\prime}(\tau) \phi(\tau) d \tau,
\end{aligned}
$$

from which we obtain that

$$
\begin{aligned}
k^{2} x(t)^{2} & \leq 2 \int_{a}^{t} x^{\prime}(\tau) \phi(\tau) d \tau, \\
M^{2} & \leq \frac{2}{k^{2}} M \phi(t) .
\end{aligned}
$$

Hence, we get that $|x(t)| \leq K \phi(t)$, where $K=\frac{2}{k^{2}}$. It is easy to see that, $y\left(t_{0}\right) \equiv 0$ is a solution of the differential equation (1.2) with (1.4) such that $\left|x(t)-y\left(t_{0}\right)\right| \leq$ $K \phi(t)$. Hence by the virtue of Definition 2.4, the differential equation (1.2) has the Hyers-Ulam-Rassias stability.

Finally, we would like to prove the Hyers-Ulam-Rassias stability of the general differential equation of FV (1.3) with initial conditions (1.4).

Theorem 4.3. If $x: I \rightarrow \mathbb{R}$ be a twice continuously differentiable function such that $\left|x^{\prime}(t)\right| \leq|x(t)|$ with $\phi(t)$, where $\phi: \mathbb{R} \rightarrow[0, \infty)$ satisfies the differential inequality

$$
\left|x^{\prime \prime}(t)+2 l x^{\prime}(t)+k^{2} x(t)-L \sin p t\right| \leq \phi(t)
$$

with initial conditions (1.4) then the differential equation (1.3) has the Hyers-UlamRassias stability with respect to $\phi(t)$ such that $\phi(a)=0$. 
Proof. Assume that $x \in C^{2}(I)$ is such that $\left|x^{\prime}(t)\right| \leq|x(t)|$ with $\phi: \mathbb{R} \rightarrow[0, \infty)$ satisfies the differential inequality (4.3) with initial conditions (1.4) and define $M=$ $\max _{t \in I}|x(t)|$. Then by the inequality (4.3), we have

$$
-\phi(t) \leq x^{\prime \prime}(t)+2 l x^{\prime}(t)+k^{2} x(t)-L \sin p t \leq \phi(t) .
$$

Multiplying the above inequality by $x^{\prime}(t)$ and then integrating $a$ to $t$, we obtain that

$$
\begin{aligned}
\int_{a}^{t}-x^{\prime}(\tau) \phi(\tau) d \tau & \leq \int_{a}^{t}\left\{x^{\prime \prime}(\tau)+2 l x^{\prime}(\tau)+k^{2} x(\tau)-L \sin p \tau\right\} x^{\prime}(\tau) d \tau \\
\leq & \int_{a}^{t} x^{\prime}(\tau) \phi(\tau) d \tau, \\
-\int_{a}^{t} x^{\prime}(t) \phi(\tau) d \tau \leq & \int_{a}^{t} x^{\prime \prime}(\tau) x^{\prime}(\tau) d \tau+2 l \int_{a}^{t} x^{\prime}(\tau)^{2} d \tau+k^{2} \int_{a}^{t} x(\tau) x^{\prime}(\tau) d \tau \\
& -L \int_{a}^{t} x^{\prime}(\tau) \sin p \tau d \tau \leq \int_{a}^{t} x^{\prime}(\tau) \phi(\tau) d \tau, \\
-2 \int_{a}^{t} x^{\prime}(\tau) \phi(\tau) d \tau \leq & x^{\prime}(t)^{2}+k^{2} x(t)^{2}+4 l \int_{a}^{t} x^{\prime}(\tau)^{2} d \tau-2 L \int_{a}^{t} x^{\prime}(\tau) \sin p \tau d \tau \\
\leq & 2 \int_{a}^{t} x^{\prime}(\tau) \phi(\tau) d \tau,
\end{aligned}
$$

from which we get that

$$
\begin{aligned}
k^{2} x(t)^{2} & \leq 2 \int_{a}^{t} x^{\prime}(\tau) \phi(\tau) d \tau-4 l \int_{a}^{t} x^{\prime}(\tau)^{2} d \tau+2 L \int_{a}^{t} x^{\prime}(\tau) \sin p \tau d \tau \\
M^{2} & \leq \frac{2}{k^{2}} M \phi(t)+\frac{4 l}{k^{2}} M^{2}(b-a)+\frac{2 L M}{k^{2}} \sin p a .
\end{aligned}
$$

Choose $\gamma=\frac{4 l(b-a)}{k^{2}}$, we get $M \leq \frac{2 \phi(t)+2 L \sin p a}{k^{2}(1-\gamma)}$, which gives

$$
|x(t)| \leq \frac{2 \phi(t)+2 L \sin p a}{k^{2}(1-\gamma)} .
$$

Hence, $|x(t)| \leq K \phi(t)$, where

$$
K \phi(t)=\frac{2 \phi(t)+2 L \sin p a}{k^{2}(1-\gamma)}
$$

for all $t \in I$. It is easy to see that, $y\left(t_{0}\right) \equiv 0$ is a solution of the differential equation (1.3) with the initial condition (1.4) such that

$$
\left|x(t)-y\left(t_{0}\right)\right| \leq K \phi(t) .
$$


Hence by the virtue of Definition 2.6, the differential equation (1.3) has the Hyers-UlamRassias stability.

\section{Conclusion}

In this paper, we have proved the Hyers-Ulam stability and Hyers-Ulam-Rassias stability of the general differential equation of the Free Damped Vibrations (FDV), Undamped Vibrations (UV) and Forced Vibrations (FV) by using initial conditions. It will be very useful to the readers to study the stability problem for various physical Phenomenon.

Acknowledgements. The authors are very grateful to the editor for the worthwhile comments. Also, we would like to thank the referees for their valuable suggestions to the improvement of this paper.

\section{REFERENCES}

[1] M. Almahalebi, A. Chahbi and S. Kabbaj, A fixed point approach to the stability of a bi-cubic functional equations in 2-Banach spaces, Palest. J. Math. 5(2) (2016), 220-227.

[2] C. Alsina and R. Ger, On some inequalities and stability results related to the exponential function, J. Inequal. Appl. 2 (1998), 373-380.

[3] T. Aoki, On the stability of the linear transformation in Banach spaces, J. Math. Soc. Japan 2 (1950), 64-66.

[4] D. G. Bourgin, Classes of transformations and bordering transformations, Bull. Amer. Math. Soc. 57 (1951), 223-237.

[5] N. Brillouet-Belluot, J. Brzdek and K. Cieplinski, On some recent developements in Ulam's type stability, Abstr. Appl. Anal. 2012 (2012), Article ID 716936, 41 pages.

[6] M. Burger, N. Ozawa and A. Thom, On Ulam stability, Israel J. Math. 193 (2013), 109-129.

[7] L. Cadariu, L. Gavruta and P. Gavruta, Fixed points and generalized Hyers-Ulam stability, Abstr. Appl. Anal. 2012 (2012), Article ID 712743, 10 pages.

[8] J. Chung, Hyers-Ulam stability theorems for Pexiders equations in the space of Schwartz distributions, Arch. Math. 84 (2005), 527-537.

[9] J. Huang and Y. Li, Hyers-Ulam stability of linear differential equations, J. Math. Anal. Appl. 426 (2015), 1192-1200.

[10] J. Huang, S. M. Jung and Y. Li, On Hyers-Ulam stability of non-linear differential equations, Bull. Korean Math. Soc. 52(2) (2015), 685-697.

[11] D. H. Hyers, On the stability of the linear functional equation, in: N. Raikhel (Ed.), Proceeding of the National Academy of Sciences of the United States of America, 27 (1941), 222-224.

[12] S. M. Jung, Hyers-Ulam-Rassias Stability of Functional Equation in Nonlinear Analysis, Springer Optimization and Its Applications 48, Springer, New York, 2011.

[13] Y. Li and Y. Shen, Hyers-Ulam stability fo linear differential equations of second order, Appl. Math. Lett. 23 (2010), 306-309.

[14] M. I. Modebei, O. O. Olaiya and I. Otaide, Generalized Hyers-Ulam stability of second order linear ordinary differential equation with initial condition, Advances in Inequalities and Applications 2014(36) (2014), 1-7.

[15] R. Murali, M. J. Rassias and V. Vithya, The general solution and stability of nonadecic functional equation in matrix normed spaces, Malaya Journal of Matematik 5(2) (2017), 416-427. 
[16] R. Murali and A. P. Selvan, On the generalized Hyers-Ulam stability of linear ordinary differential equations of higher order, International Journal of Pure and Applied Mathematics 117(12) (2017), 317-326.

[17] R. Murali and A. P. Selvan, Hyers-Ulam stability of nth order differential equation, Contemporary Studies in Discrete Mathematics (CSDM) 2(1) (2018), 45-50.

[18] R. Murali and A. P. Selvan, Hyers-Ulam-Rassias stability for the linear ordinary differential equation of third order, Kragujevac J. Math. 42(4) (2018), 579-590.

[19] M. Obloza, Connections between Hyers and Lyapunov stability of the ordinary differential equations, Rocznik Nauk.-Dydakt. Prace Matematyczne 14 (1997), 141-146.

[20] M. Obloza, Hyers stability of the linear differential equation, Rocznik Nauk.-Dydakt. Prace Matematyczne 13 (1993), 259-270.

[21] Th. M. Rassias, On the stability of the linear mapping in Banach spaces, Proc. Amer. Math. Soc. 72 (1978), 297-300.

[22] K. Ravi, R. Murali and A. P. Selvan, Ulam stability of a general nth order linear differential equation with constant coefficients, Asian Journal of Mathematics and Computer Research 11(1) (2016), 61-68.

[23] K. Ravi, R. Murali and A. P. Selvan, Hyers-Ulam stability of nth order linear differential equation with initial and boundary condition, Asian Journal of Mathematics and Computer Research 11(3) (2016), 201-207.

[24] K. Ravi, J. M. Rassias and B. V. S. Kumar, Ulam-Hyers stability of undecic functional equation in quasi-beta-normed spaces fixed point method, Tbilisi Math. J. 9(2) (2016), 83-103.

[25] K. Ravi, J. M. Rassias, S. Pinelas and S. Suresh, General solution and stability of quattuordecic functional equation in quasi-beta-normed spaces, Advances in Pure Mathematics 6 (2016), 921-941.

[26] I. A. Rus, Ulam stabilities of ordinary differential equations in Banach space, Carpathian J. Math. 26(1) (2010), 103-107.

[27] S. Takahasi, T. Miura and S. Miyajima, On the Hyers-Ulam stability of the Banach space valued differential equation $y^{\prime}(t)=\lambda y(t)$, Bull. Korean Math. Soc. 39 (2002), 309-315.

[28] S. M. Ulam, A Collection of Mathematical Problems, Interscience Publishers, New York, 1960.

[29] T. Z. Xu, On the stability of Multi-Jensens mappings in $\beta$-normed spaces, Appl. Math. Lett. 25(11) (2012), 1866-1870.

[30] A. Zada, O. Shah and R. Shah, Hyers-Ulam stability of non-autonomous systems in terms of boundedness of cauchy problems, Appl. Math. Comput. 271 (2015), 512-518.

${ }^{1}$ PG and Research Department of Mathematics,

Sacred Heart College (Autonomous),

Tirupattur - 635 601, Vellore Dt. TAmil Nadu, India

Email address: shcrmurali@yahoo.co.in

Email address: selvaharry@yahoo.com 